\title{
Design and synthesis of thrombin substrates with modified kinetic parameters
}

Citation for published version (APA):

Rijkers, D. T. S., Wielders, S. J. H., Tesser, G. I., \& Hemker, H. C. (1995). Design and synthesis of thrombin substrates with modified kinetic parameters. Thrombosis Research, 79(5/6), 491-499. https://doi.org/10.1016/0049-3848(95)00139-I

Document status and date:

Published: 01/09/1995

DOI:

10.1016/0049-3848(95)00139-I

Document Version:

Publisher's PDF, also known as Version of record

\section{Please check the document version of this publication:}

- A submitted manuscript is the version of the article upon submission and before peer-review. There can be important differences between the submitted version and the official published version of record.

People interested in the research are advised to contact the author for the final version of the publication, or visit the DOI to the publisher's website.

- The final author version and the galley proof are versions of the publication after peer review.

- The final published version features the final layout of the paper including the volume, issue and page numbers.

Link to publication

\footnotetext{
General rights rights.

- You may freely distribute the URL identifying the publication in the public portal. please follow below link for the End User Agreement:

www.umlib.nl/taverne-license

Take down policy

If you believe that this document breaches copyright please contact us at:

repository@maastrichtuniversity.nl

providing details and we will investigate your claim.
}

Copyright and moral rights for the publications made accessible in the public portal are retained by the authors and/or other copyright owners and it is a condition of accessing publications that users recognise and abide by the legal requirements associated with these

- Users may download and print one copy of any publication from the public portal for the purpose of private study or research.

- You may not further distribute the material or use it for any profit-making activity or commercial gain

If the publication is distributed under the terms of Article $25 \mathrm{fa}$ of the Dutch Copyright Act, indicated by the "Taverne" license above, 
0049-3848(95)00139-5

\title{
DESIGN AND SYNTHESIS OF THROMBIN SUBSTRATES WITH MODIFIED KINETIC PARAMETERS
}

\author{
Dirk T.S. Rijkers ${ }^{1,2}$, Simone J.H. Wielders ${ }^{1}$, Godefridus I. Tesser ${ }^{2}$ \\ and H.Coenraad Hemker ${ }^{1}$ \\ ${ }^{1}$ University of Limburg, Faculty of Medicine, Department of Biochemistry, P.O. Box 616, \\ 6200 MD Maastricht, The Netherlands \\ ${ }^{2}$ Catholic University of Nijmegen, Department of Organic Chemistry, Toernooiveld, \\ 6525 ED Nijmegen, The Netherlands
}

(Received 14 March 1995 by Editor J. Stürzebecher; revised/accepted 4 July 1995)

\begin{abstract}
For the continuous registration of thrombin formation in plasma (1), selective thrombin substrates are required, that show moderate binding affinities (high $\mathrm{K}_{\mathrm{m}}$ ) and low turnover numbers (low $\left.\mathrm{k}_{\text {cat }}\right)$. Previously we have used SQ68 $\left(\mathrm{CH}_{3} \mathrm{O}-\mathrm{CO}-\mathrm{CH}_{2}-\mathrm{CO}-\right.$ Aib-Arg-pNA) for this purpose. In order to find more substrates suitable for this application, we synthesized a series of 25 peptide $p$-nitroanilides. As lead structures SQ68 and S2238 (H-D-Phe-Pip-Arg-pNA) were used. By introduction of specific structure modifications we tried to alter the kinetic data in the required direction. The modifications were designed on basis of existing knowledge on the structure of the thrombin active-site and its surroundings. We indeed obtained a number of substrates with the kinetic constants in the desired range.
\end{abstract}

For the continuous registration of thrombin generation and the determination of the endogenous thrombin potential (ETP: area under the thrombin generation curve) it is necessary to dispose of specific thrombin substrates that are converted at a slow rate so as not to be exhausted when added to clotting plasma (1). The suitable substrate that is now available (Serbio SQ68), was found by accident. Published modeling and X-ray analyses of the thrombin molecule offer insight into the three-dimensional form of its active-site and suggest modifications of existing thrombin substrates that may serve to tune the kinetic parameters in the required direction. In this article we report the results of a synthetic search for specific, slow reacting thrombin substrates

Current research focusses on H-D-Phe-Pip-Arg-pNA (S2238) as a lead structure in the development of highly specific thrombin inhibitors. Derivatives of this structure containing aldehyde- (2), chloromethylketone- (3), aminoboronic acid- (4), ketomethylene- (5) and aminophosphonic acid- (6) functions were described as potent and specific transition-state

$\overline{\text { Key words: }}$ chemical synthesis, chromogenic substrates, thrombin substrates.

Abbreviations: Abbreviations used for amino acids and for the designations of peptides follow the rules of the IUPAC-IUB Joint Commission on Biochemical Nomenclature in Eur. J. Biochem. 138, 9-37, 1984

Corresponding author: Dr. H.C. Hemker, University of Limburg, Faculty of Medicine, Department of Biochemistry, P.O. Box 616, 6200 MD Maastricht, The Netherlands, Phone: +3143881675 , Fax: +3143670988 . 
analogue inhibitors of thrombin. All these inhibitors contain an arginine in the P1 position corresponding to the primary specificity of thrombin.

Recent publications showed that inhibitors derived from H-D-Phe-Pip-Arg that, at position P1, contain an amino acid with a neutral side chain show enhanced selectivity towards thrombin. Also, substances in which the positively charged side chain of arginine is replaced by a neutral side chain, containing aldehyde- (7), aminoboronic acid- (8) and aminophosphonic acid- (9) functions bind tightly to thrombin. This result is somewhat surprising, because it was believed that electrostatic interaction between the positively charged guanidino group of inhibitors and substrates, and the negatively charged Asp189 residue in the primary specificity pocket of thrombin is essential for complex formation. The crystal structure of human $\alpha$-thrombin complexed with $\mathrm{H}-\mathrm{D}$-Phe-Pro-Arg- $\mathrm{CH}_{2} \mathrm{Cl}$, however, showed that the active-site consists of two important binding regions: the hydrophobic binding pocket, $\mathrm{S} 2$ subsite, which interacts preferentially with proline residues, and the aryl-binding pocket (S3) in which aromatic stacking interactions (D-phenylalanine) are of major importance for strong binding affinity (10-11). The fact that inhibitors containing the H-D-Phe-Pro sequence with a modified P1 residue still bind to thrombin can be explained in terms of these binding interactions. Also, the inhibitors containing amino acids with neutral side chains at the $P_{1}$ position show enhanced selectivity towards thrombin compared to other serine proteases with an arginine as primary determinant of specificity. Comparison of the S1 binding pockets of thrombin, trypsin and plasmin showed that the $\mathrm{S} 1$ binding pocket of thrombin is the most hydrophobic one. This explains why a neutral side chain at $P 1$ would be better accommodated by thrombin than by trypsin or plasmin. The improvement of selectivity is paid by a decrease of potency as a result of a loss in electrostatic binding interactions with Asp 189.

These findings motivated us to try and synthesize pNA-substrates with maintenance of the H-DPhe-Pip(Pro) sequence and in which the P1 amino acid residue is varied, see table I.

Another starting point was $\mathrm{CH}_{3} \mathrm{O}-\mathrm{CO}-\mathrm{CH}_{2}$-CO-Aib-Arg-pNA (SQ68) (12). At position $\mathrm{P} 2$ and $\mathrm{P}_{3}$, this substrate has not the optimal structural elements for interaction with thrombin. We started an investigation into a structure-activity relationship to obtain substrates selective for thrombin, in which a dipeptide is the smallest determinant of selectivity, the case being modified at its N-terminus to mimic a $\mathrm{P} 3$ residue. From modeling studies (13-14) and homology modelbuilding (15) it has been concluded that the $\beta$-naphtalenesulfonyl function interacts with thrombin in a highly specific manner, whereas interaction with other serine proteases is minimal. The $p$ toluenesulfonyl group showed the same binding interactions as the $\beta$-naphthalenesulfonyl moiety, albeit to a somewhat lesser extent (16). These findings were confirmed by X-ray studies of thrombin-complexes formed with active-site directed inhibitors containing these structural elements $(17-18)$. We used both functions to arrive at more selective thrombin substrates.

\section{EXPERIMENTAL PROCEDURES}

\section{General}

${ }^{1} \mathrm{H}$ Nuclear magnetic resonance (NMR) spectra were recorded on Bruker AM 100 and Bruker AM 400 spectrometers (Palo Alto, USA). As an internal standard the residual solvent peak was used. Chemical shifts are given in part per million (ppm). Optical rotations were measured on a Perkin Elmer 241 polarimeter in a $10 \mathrm{~cm}$ cuvette at room temperature. Melting points were determined with a Büchi melting point apparatus (Tottoli). Thin layer chromatography (TLC) was performed on Merck Silicagel $60 \mathrm{~F}_{254}$ plates (Darmstadt, Germany), and column chromatography on Merck Kieselgel 60, 70-230 Mesh ASTM. Spots were detected by IJVfluorescence quenching, ninhydrine (free amino functions), chlorine/TDM (4,4'-tetramethyldiaminodiphenylmethane, NH groups), Barton's- (hydrazides) and Sakaguchi's (arginine residues) reagent. Methanol was refluxed on magnesium turnings for two hours, distilled and stored on $3 \AA$ molsieves. Pyridine was distilled over $\mathrm{KOH}$ pellets and stored on $4 \AA$ molsieves. tert.Butyloxycarbonyl (Boc)-amino acids were synthesized by the method of Schnabel (19). Amino acid $p$-nitroanilides were prepared as described by us $(20)$. 


\section{TABLE I}

\section{Substrates of the Type H-D-Phe-Y-X-pNA and Y-X-Arg-pNA with S2238 respectively SQ68 as Lead Structures.}

\begin{tabular}{rrr}
\multicolumn{2}{c}{ H-D-Phe-Y-X-pNA } & \\
Y & X & Code \\
Pip & Lys & $\mathbf{1 a}$ \\
Pip & Orn & $\mathbf{1 b}$ \\
Pip & Glu & $\mathbf{1 c}$ \\
Pip & Nle & $\mathbf{1 d}$ \\
& & \\
Pro & Lys & $\mathbf{2 a}$ \\
Pro & Nle & $\mathbf{2 b}$ \\
& & \\
& Y-X-Arg-pNA & \\
Y & X & Code \\
& & \\
MZ & Gly & $\mathbf{3 a}$ \\
MMZ & Gly & $\mathbf{3 b}$ \\
DMMZ & Gly & $\mathbf{3 c}$ \\
DEMZ & Gly & $\mathbf{3 d}$ \\
Nas & Gly & $\mathbf{3 e}$ \\
Tos & Gly & $\mathbf{3 f}$ \\
& & \\
MZ & Ala & $\mathbf{4 a}$ \\
MMZ & Ala & $\mathbf{4 b}$ \\
DMMZ & Ala & $\mathbf{4 c}$ \\
MZ & & \\
& D-Ala & $\mathbf{5 a}$ \\
MZ & & \\
& Val & $\mathbf{6 a}$ \\
MZ & & \\
& D-Val & $\mathbf{7 a}$ \\
& &
\end{tabular}

\author{
Derived from \\ H-D-Phe-Pip-Arg-pNA \\ Code \\ 1 \\ H-D-Phe-Pro-Arg-pNA \\ 2 \\ Derived from Code \\ H-Gly-Arg-pNA \\ 3
}

H-Ala-Arg-pNA

4

$\begin{array}{rr}\text { H-D-Ala-Arg-pNA } & 5 \\ \text { H-Val-Arg-pNA } & 6\end{array}$

H-D-Val-Arg-pNA

7

Unprotected amino functions are suitably protonated $(\mathrm{HCl})$ to give neutral solutions in plain water.

Malonic acid and dimethylmalonic acid were converted to their corresponding dimethyl esters by heating the dicarboxylic acid with an excess of methanol in the presence of concentrated sulfuric acid. Dimethyl methylmalonate was prepared by generating the mono-sodio derivative of dimethylmalonate which reacts with methyl iodide via a $\mathrm{S}_{\mathrm{N}} 2$ mechanism. The corresponding half esters were prepared by controlled partial hydrolysis of the diester with one equivalent of potassium hydroxide. The monomethyl ester of diethylmalonic acid was obtained in a direct way by refluxing the acid in an excess of methanol with sulfuric acid as catalyst. Dimethylmalonic acid and L-pipecolic acid were from Janssen Chimica (Geel, Belgium), diethylmalonic acid was from Aldrich (Bornem, Belgium). TDM, naphthalene-2-sulfonyl chloride, toluene-4-sulfonyl chloride and D-alanine were obtained from Fluka (Buchs, Switzerland). Boc-Nle-OH, and FmocOrn(Boc)-OH were from Bachem (Bubendorf, Switzerland). D-Phenylalanine was a generous gift of Dr. J. Kamphuis (DSM Research, Geleen, The Netherlands). 


\begin{abstract}
Syntheses
Boc-D-Phe-Pip- $\mathrm{N}_{2} \mathrm{H}_{3}$ :

To a solution of Boc-D-Phe-OH $(19.88 \mathrm{~g}, 75 \mathrm{mmol})$ in EtOAc $(350 \mathrm{~mL})$ was added HCl.H-PipOMe (21) (13.46 g, $75 \mathrm{mmol})$, HOBt (12.62 g, $82.5 \mathrm{mmol})$ and NMM (8.75 mL, $78.7 \mathrm{mmol})$. The obtained suspension was cooled on ice and DCC $(16.22 \mathrm{~g}, 78.7 \mathrm{mmol})$ was added. After stirring for two hrs at $0^{\circ} \mathrm{C}$ and $16 \mathrm{hrs}$ at room temperature, DCU was filtered off. The EtOAc solution was washed with $2 \mathrm{~N} \mathrm{KHSO}_{4}, \mathrm{H}_{2} \mathrm{O}$, saturated $\mathrm{NaHCO}_{3}$ and brine (four times $50 \mathrm{~mL}$ each). The EtOAc layer was dried $\left(\mathrm{Na}_{2} \mathrm{SO}_{4}\right)$, filtered and evaporated in vacuo, yielding a clear oil, yield: $21.52 \mathrm{~g}(74 \%)$. This product was checked by TLC on purity and directly used for further synthesis $\left(\mathrm{R}_{\mathrm{f}}\left(\mathrm{CH}_{2} \mathrm{Cl}_{2} / \mathrm{MeOH} 9: 1 \mathrm{v} / \mathrm{v}\right): 0.87\right)$. The protected dipeptide methyl ester was dissolved in $\mathrm{MeOH}(150 \mathrm{~mL})$ and $\mathrm{N}_{2} \mathrm{H}_{4} \cdot \mathrm{H}_{2} \mathrm{O}(13.5 \mathrm{~mL}, 278 \mathrm{mmol})$ was added. This reaction mixture was left for three days at room temperature. The formed precipitate was filtered and dried; yield: $15.01 \mathrm{~g}(70 \%), \mathrm{R}_{\mathrm{f}}\left(\mathrm{CH}_{2} \mathrm{Cl}_{2} / \mathrm{MeOH} 9: 1 \mathrm{v} / \mathrm{v}\right): 0.54,[\alpha]_{\mathrm{D}}=-77.1^{\circ} \mathrm{c}=0.98 \mathrm{DMF}, \mathrm{mp}$ : $202-204^{\circ} \mathrm{C},{ }^{1} \mathrm{H}$ NMR $\left(\mathrm{CD}_{3} \mathrm{OD}\right): \delta=1.15-1.38\left(\mathrm{~m}, 6 \mathrm{H}, \mathrm{CH}_{2}-\mathrm{Pip}(3 \times 2 \mathrm{H})\right) ; 1.44(\mathrm{~s}, 9 \mathrm{H}$, Boc); $3.08 / 3.18\left(\mathrm{dm}, 2 \mathrm{H}, \beta-\mathrm{CH}_{2}-\mathrm{Phe}\right) ; 3.28\left(\mathrm{~m}, 2 \mathrm{H}, \mathrm{N}-\mathrm{CH}_{2}-\mathrm{Pip}\right) ; 4.58(\mathrm{~m}, 1 \mathrm{H}, \alpha-\mathrm{CH}-\mathrm{Pip}) ; 4.74(\mathrm{~m}$, $1 \mathrm{H}, \alpha$-CH-Phe); 7.20-7.33 (m, 5H, arom Phe).
\end{abstract}

Boc-D-Phe-Pip-X-pNA

a) $X=\operatorname{Orn}(B o c)$ :

Boc-D-Phe-Pip- $\mathrm{N}_{2} \mathrm{H}_{3}(0.39 \mathrm{~g}, 1.0 \mathrm{mmol})$ was dissolved in DMF $(10 \mathrm{~mL})$ and cooled to $-20^{\circ} \mathrm{C}$. To this solution was added $2.3 \mathrm{M} \mathrm{HCl} /$ EtOAc $(1.20 \mathrm{~mL}, 2.75 \mathrm{mmol})$ followed by tert.-BuONO $\left(0.114 \mathrm{~mL}, 1.20 \mathrm{mmol}\right.$ ). This reaction mixture was stirred for $15 \mathrm{~min}$ at $-20^{\circ} \mathrm{C}$. (After this period the azide-formation was complete.) The acid solution was neutralized by adding DIPEA ( 0.48 $\mathrm{mL}, 2.77 \mathrm{mmol})$. H-Orn(Boc)-pNA $(0.328 \mathrm{~g}, 1.0 \mathrm{mmol})$ was added. This reaction mixture was kept on neutrality by adding DIPEA at regular time intervals. The obtained reaction medium was allowed to react during $16 \mathrm{hrs}$ at $0^{\circ} \mathrm{C}$. After this period the DMF phase was removed in vacuo and the oily residue was dissolved in EtOAc $(10 \mathrm{~mL})$. The EtOAc solution was washed with $\mathrm{H}_{2} \mathrm{O}, 2 \mathrm{~N} \mathrm{KHSO}, \mathrm{H}_{2} \mathrm{O}$ saturated $\mathrm{NaHCO}_{3}$ and brine (three times $5 \mathrm{~mL}$ each). The EtOAc solution was dried $\left(\mathrm{Na}_{2} \mathrm{SO}_{4}\right)$, filtered and evaporated in vacuo. Boc-D-Phe-Pip-Orn(Boc)-pNA was purified by column chromatography on silica gel with $\mathrm{CH}_{2} \mathrm{Cl}_{2} / \mathrm{MeOH} 98: 2 \mathrm{v} / \mathrm{v}$ as eluens. Yield: $0.525 \mathrm{~g}(74 \%), \mathrm{R}_{\mathrm{f}}\left(\mathrm{CH}_{2} \mathrm{Cl}_{2} / \mathrm{MeOH} 98: 2 \mathrm{v} / \mathrm{v}\right): 0.22,[\alpha]_{\mathrm{D}}=-64.8^{\circ} \mathrm{c}=0.13 \mathrm{MeOH},{ }^{1} \mathrm{H}$ NMR $\left(\mathrm{CD}_{3} \mathrm{OD}\right): \delta=1.26-1.48\left(\mathrm{bm}, 6 \mathrm{H}, \mathrm{CH}_{2}-\mathrm{Pip}(3 \times 2 \mathrm{H})\right.$ ); $1.34(\mathrm{~s}, 9 \mathrm{H}, \mathrm{Boc}) ; 1: 41$ (s, 9H, Boc); $1.56 / 1.65\left(\mathrm{dm}, 2 \mathrm{H}, \gamma-\mathrm{CH}_{2}-\mathrm{Orn}\right) ; 1.78 / 1.99\left(\mathrm{dm}, 2 \mathrm{H}, \beta-\mathrm{CH}_{2}-\mathrm{Orn}\right) ; 2.97\left(\mathrm{~m}, 2 \mathrm{H}, \beta-\mathrm{CH}_{2}-\right.$ Phe); 3.07 (m, 4H, $\delta$ - $\mathrm{CH}_{2}$-Orn/N-CH $\left.2-\mathrm{Pip}\right) ; 4.48$ (m, 2H, $\alpha$-CH-Orn/ $\alpha$-CH-Pip); 4.79 (m, 1H, $\alpha-$ CH-Phe); 7.25-7.32 (m, 5H, arom Phe); 7.86/7.88-8.18/8.20 (dd, 4H, arom pNA).

b) $X=G l u\left(O^{t} B u\right)$

Obtained as described for a).

Yield: $0.422 \mathrm{~g}(62 \%), \mathrm{R}_{\mathrm{f}}\left(\mathrm{CH}_{2} \mathrm{Cl}_{2} / \mathrm{MeOH} 98: 2 \mathrm{v} / \mathrm{v}\right): 0.44,[\alpha]_{\mathrm{D}}=-78.5^{\circ} \mathrm{c}=0.33 \mathrm{MeOH},{ }^{1} \mathrm{H}$ NMR (CD $\mathrm{OD}): \delta=1.26-1.54\left(\mathrm{bm}, 8 \mathrm{H}, \beta-\mathrm{CH}_{2}-\mathrm{Glu} / \mathrm{CH}_{2}-\mathrm{Pip}(3 \mathrm{x} 2 \mathrm{H})\right) ; 1.33\left(\mathrm{~s}, 9 \mathrm{H}, \mathrm{O}^{\mathrm{B} u}\right) ; 1.43$ (s, 9H, Boc); 2.38 (m, 2H, $\left.\gamma-\mathrm{CH}_{2}-\mathrm{Glu}\right) ; 2.96\left(\mathrm{~m}, 2 \mathrm{H}, \beta-\mathrm{CH}_{2}-\mathrm{Phe}\right) ; 3.08$ (m, 2H, N-CH $-\mathrm{Pip}$ ); 4.53 (dd, 1H, $\alpha$-CH-Glu); 4.59 (m, 1H, $\alpha$-CH-Pip); 4.82 (m, 1H, $\alpha-C H-P h e) ; 7.26-7.33 ~(m, 5 H$, arom Phe); 7.89/7.91-8.20/8.22 (dd, $4 \mathrm{H}$, arom pNA).

$n H C l . H-D-P h e-P i p-X-p N A$

The protected tripeptide was dissolved in $\mathrm{AcOH}(1 \mathrm{mmol}$ in $5 \mathrm{~mL}$ ). To the solution $2.3 \mathrm{M}$ $\mathrm{HCl} / \mathrm{EtOAc}(10 \mathrm{~mL}$ for $1 \mathrm{mmol})$ was added and the reaction mixture was stirred until deprotection was complete. The acid was quenched with tert. $\mathrm{BuOH}(10 \mathrm{~mL})$ and evaporated in vacuo. The residue was coevaporated with tert. $\mathrm{BuOH}$ (twice) and with $\mathrm{MeOH}$ (once). The residue was dissolved in $\mathrm{H}_{2} \mathrm{O}$ and lyophilized. Crude tripeptides were purified by counter current distribution with $\mathrm{BuOH} / \mathrm{AcOH} / \mathrm{H}_{2} \mathrm{O} 4: 1: 5 \mathrm{v} / \mathrm{v} / \mathrm{v}$ as solvent system.

a) $n=2, X=\operatorname{Orn}(\mathbf{1 b})$ :

Yield: $0.315 \mathrm{~g}(96 \%), \mathrm{R}_{\mathrm{f}}\left(\mathrm{BuOH} / \mathrm{AcOH} / \mathrm{H}_{2} \mathrm{O} 4: 1: 1 \mathrm{v} / \mathrm{v} / \mathrm{v}\right): 0.42(\mathrm{~K}=0.71),[\alpha]_{\mathrm{D}}=-96.7^{\circ} \mathrm{c}=$ $0.18 \mathrm{MeOH},{ }_{\mathrm{H}} \mathrm{NMR}\left(\mathrm{CD}_{3} \mathrm{OD}\right): \delta=1.29-1.49\left(\mathrm{~m}, 6 \mathrm{H}, \mathrm{CH}_{2}-\mathrm{Pip}(3 \times 2 \mathrm{H})\right) ; 1.62-1.77(\mathrm{dm}, 2 \mathrm{H}$, $\left.\gamma-\mathrm{CH}_{2}-\mathrm{Orn}\right) ; 2.00-2.15\left(\mathrm{dm}, 2 \mathrm{H}, \beta-\mathrm{CH}_{2}-\mathrm{Orn}\right) ; 2.98\left(\mathrm{~m}, 6 \mathrm{H}, \beta-\mathrm{CH}_{2}-\mathrm{Phe} / \mathrm{N}-\mathrm{CH}_{2}-\mathrm{Pip} / \delta-\mathrm{CH}_{2}-\mathrm{Orn}\right) ;$ $4.34(\mathrm{~m}, 1 \mathrm{H}, \alpha-\mathrm{CH}-\mathrm{Pip}) ; 4.46(\mathrm{~m}, 1 \mathrm{H}, \alpha-\mathrm{CH}-\mathrm{Orn}) ; 4.57$ (m, 1H, $\alpha-\mathrm{CH}-\mathrm{Phe}) ; 7.23-7.35$ (m, 5H, arom Phe); 7.85/7.87-8.20/8.22 (dd, $4 \mathrm{H}$, arom $\mathrm{pNA}$ ). 
b) $\boldsymbol{n}=1, X=$ Glu $(\mathbf{l c})$ :

Yield: $0.158 \mathrm{~g}(64 \%), \mathrm{R}_{\mathrm{f}}\left(\mathrm{BuOH} / \mathrm{AcOH} / \mathrm{H}_{2} \mathrm{O} 4: 1: 1 \mathrm{v} / \mathrm{v} / \mathrm{v}\right): 0.67(\mathrm{~K}=5.15),[\alpha]_{\mathrm{D}}=-107.4^{\circ} \mathrm{c}=$ $0.22 \mathrm{MeOH}, \mathrm{H} \mathrm{NMR}\left(\mathrm{CD}_{3} \mathrm{OD}\right): \delta=1.11-1.35\left(\mathrm{~m}, 6 \mathrm{H}, \mathrm{CH}_{2}-\mathrm{Pip}(3 \times 2 \mathrm{H}) ; 2.03\left(\mathrm{~m}, 2 \mathrm{H}, \beta-\mathrm{CH}_{2}-\right.\right.$ Glu); 2.26/2.42 (dm, 2H, $\left.\gamma-\mathrm{CH}_{2}-\mathrm{Glu}\right) ; 2.82 / 3.55$ (dm, 2H, N-CH $\left.-\mathrm{Pip}\right) ; 3.11 / 3.24$ (dm, 2H, $\beta-$ $\mathrm{CH}_{2}$-Phe); 4.17 (m, 1H, $\left.\alpha-\mathrm{CH}-\mathrm{Glu}\right) ; 4.52$ (m, 1H, $\alpha$-CH-Pip); 4.82 (m, 1H, $\alpha-\mathrm{CH}-\mathrm{Phe}$ ); $7.26-$ $7.41(\mathrm{~m}, 5 \mathrm{H}$, arom Phe); 7.84/7.86-8.19/8.22 (dd, $4 \mathrm{H}$, arom $\mathrm{pNA})$

Boc-X-Arg-pNA.HCl

a) $X=$ Gly:

Boc-Gly-OH (1.75 g, $10 \mathrm{mmol})$, 2HCl.H-Arg-pNA (3.67 g, $10 \mathrm{mmol})$, HOBt $(1.60 \mathrm{~g}, 10.1$ mmol) and NMM (1.10 mL, $10.0 \mathrm{mmol})$ were dissolved in DMF $(75 \mathrm{~mL})$. This reaction mixture was cooled on ice and DCC (2.16 g, $10.5 \mathrm{mmol}, 1.05 \mathrm{eq}$.) was added. After stirring for $1 \mathrm{hr}$ at $0^{\circ} \mathrm{C}$ and $16 \mathrm{hrs}$ at room temperature, DCU was filtered off and DMF was removed under reduced pressure. The residue was purified by counter current distribution with $\mathrm{BuOH} / \mathrm{AcOH} / \mathrm{H}_{2} \mathrm{O} 4: 1: 5$ $\mathrm{v} / \mathrm{v} / \mathrm{v}(\mathrm{K}=1.84)$ as the solvent system. Pure product was lyophilized from AcOH. Yield: $4.29 \mathrm{~g}$ $(88 \%), \mathrm{R}_{\mathrm{f}}\left(\mathrm{BuOH} / \mathrm{AcOH} / \mathrm{H}_{2} \mathrm{O} 4: 1: 1 \mathrm{v} / \mathrm{v} / \mathrm{v}\right): 0.64,[\alpha]_{\mathrm{D}}=-36.8^{\circ} \mathrm{c}=0.63 \mathrm{MeOH}$.

b) $X=V a l$ :

Obtained as described for a).

Yield: $2.36 \mathrm{~g}(89 \%), \mathrm{R}_{\mathrm{f}}\left(\mathrm{BuOH} / \mathrm{AcOH} / \mathrm{H}_{2} \mathrm{O} 4: 1: 1 \mathrm{v} / \mathrm{v} / \mathrm{v}\right): 0.69(\mathrm{~K}=2.11),[\alpha]_{\mathrm{D}}=-44.4^{\circ} \mathrm{c}=0.59$ $\mathrm{MeOH},{ }^{1} \mathrm{H}$ NMR $\left(\mathrm{CD}_{3} \mathrm{OD}\right): \delta=0.94-0.98\left(\mathrm{dd}, 6 \mathrm{H}, \gamma-\mathrm{CH}_{3} / \gamma^{\prime}-\mathrm{CH}_{3}-\mathrm{Val}\right) ; 1.44$ (s, 9H, Boc); 1.70 (m, 1H, $\beta-\mathrm{CH}-\mathrm{Val}) ; 1.82\left(\mathrm{~m}, 2 \mathrm{H}, \gamma-\mathrm{CH}_{2}-\mathrm{Arg}\right) ; 2.03\left(\mathrm{~m}, 2 \mathrm{H}, \beta-\mathrm{CH}_{2}-\mathrm{Arg}\right) ; 3.23\left(\mathrm{~m}, 2 \mathrm{H}, \delta-\mathrm{CH}_{2}-\right.$ Arg); $3.88(\mathrm{~d}, 1 \mathrm{H}, \alpha-\mathrm{CH}-\mathrm{Val}) ; 4.56(\mathrm{~m}, 1 \mathrm{H}, \alpha-\mathrm{CH}-\mathrm{Arg}) ; 7.84 / 7.86-8.20 / 8.22$ (dd, $4 \mathrm{H}$, arom pNA)

2HCl.H-Val-Arg-pNA (6):

As described for nHCl.H-D-Phe-Pip-X-pNA.

Yield: $0.401 \mathrm{~g}(86 \%), \mathrm{R}_{\mathrm{f}}\left(\mathrm{BuOH} / \mathrm{AcOH} / \mathrm{H}_{2} \mathrm{O} 4: 1: 1 \mathrm{v} / \mathrm{v} / \mathrm{v}\right): 0.40,[\alpha]_{\mathrm{D}}=-5.7^{\circ} \mathrm{c}=0.21 \mathrm{MeOH},{ }^{1} \mathrm{H}$ NMR (CD 30 OD): $\delta=1.05 / 1.07-1.08 / 1.10\left(\mathrm{dd}, 6 \mathrm{H}, \gamma-\mathrm{CH}_{3} / \gamma^{\prime}-\mathrm{CH}_{3}-\mathrm{Val}\right) ; 1.70-1.89\left(\mathrm{~m}, 3 \mathrm{H}, \gamma-\mathrm{CH}_{2}-\right.$ $\mathrm{Arg} / \beta-\mathrm{CH}-\mathrm{Val}$ ); $1.96 / 2.25\left(\mathrm{dm}, 2 \mathrm{H}, \beta-\mathrm{CH}_{2}-\mathrm{Arg}\right.$ ); $3.26\left(\mathrm{~m}, 2 \mathrm{H}, \delta-\mathrm{CH}_{2}-\mathrm{Arg}\right) ; 3.82$ (d, $1 \mathrm{H}, \alpha-\mathrm{CH}-$ $\mathrm{Val}) ; 4.58(\mathrm{~m}, 1 \mathrm{H}, \alpha-\mathrm{CH}-\mathrm{Arg}) ; 7.85 / 7.87-8.20 / 8.22$ (dd, $4 \mathrm{H}$, arom pNA).

$R-X-A r g-p N A . H C l$

The monomethyl ester of the malonic acid derivative was coupled with DCC/HOBt in DMF with $2 \mathrm{HCl} . \mathrm{H}-\mathrm{X}$-Arg-pNA in the presence of DIPEA for 1 hour at $0^{\circ} \mathrm{C}$ and $16 \mathrm{hrs}$ at room temperature. After filtration, the clear DMF phase was evaporated in vacuo. The residue was purified by counter current distribution with the solvent system: $\mathrm{BuOH} / \mathrm{AcOH} / \mathrm{H}_{2} \mathrm{O}$ 4:1:5 v/v/v. Pure product was lyophilized from $\mathrm{H}_{2} \mathrm{O}$.

$R=D E M Z, X=G l y(3 d)$ :

Yield: $0.398 \mathrm{~g}(74 \%), \mathrm{R}_{\mathrm{f}}\left(\mathrm{BuOH} / \mathrm{AcOH} / \mathrm{H}_{2} \mathrm{O} 4: 1: 1 \mathrm{v} / \mathrm{v} / \mathrm{v}\right): 0.57(\mathrm{~K}=2.31),[\alpha]_{\mathrm{D}}=-29.8^{\circ} \mathrm{c}=0.27$ $\mathrm{MeOH},{ }^{1} \mathrm{H}$ NMR $\left(\mathrm{D}_{2} \mathrm{O}\right): \delta=0.85\left(\mathrm{t}, 6 \mathrm{H}, \mathrm{CH}_{3}\right.$-diethylmalonic acid $\left.(2 \times 3 \mathrm{H})\right) ; 1.59(\mathrm{~m}, 4 \mathrm{H}, \beta-$ $\left.\mathrm{CH}_{2} / \gamma-\mathrm{CH}_{2}-\mathrm{Arg}\right) ; 1.92\left(\mathrm{q}, 4 \mathrm{H}, \sim \mathrm{CH}_{2} \sim\right.$ diethylmalonic acid $\left.(2 \times 2 \mathrm{H})\right) ; 3.19\left(\mathrm{~m}, 2 \mathrm{H}, \delta-\mathrm{CH}_{2}-\mathrm{Arg}\right)$; 3.79 (s, 3H, OCH 3 ); 3.92 (s, 2H, $\mathrm{CH}_{2}-\mathrm{Gly}$ ); 4.24 (m, 1H, $\alpha-\mathrm{CH}-\mathrm{Arg}$ ); 7.25/7.32-7.64/7.73 (dd, $4 \mathrm{H}$, arom pNA).

Chromogenic substrates: The substrates were dissolved in distilled water to obtain $10 \mathrm{mM}$ stock solutions. The concentration was determined at $316 \mathrm{~nm}$ using a molar extinction coefficient of $12,500 \mathrm{Lmol}^{-1} \mathrm{~cm}^{-1}$. Stock solutions were stored in the dark at $4^{\circ} \mathrm{C}$.

Enzyme preparations: Pure human $\alpha$-thrombin and human factor Xa samples were generous gifts of Dr. T. Lindhout (University of Limburg, Maastricht, The Netherlands).

Kinetic parameters: The hydrolysis experiments were run in buffer $\mathrm{A}(0.05 \mathrm{M}$ Tris- $\mathrm{HCl}, 0.1 \mathrm{M}$ $\mathrm{NaCl}, \mathrm{pH}=7.35$ containing $0.5 \mathrm{~g} / \mathrm{L}$ bovine serum albumin (Sigma, Bornem, Belgium)) at $37^{\circ} \mathrm{C}$. The liberation of $p$-nitroaniline was monitored at $405 \mathrm{~nm}$ in a dual wavelength $(405-546 \mathrm{~nm})$ spectrophotometer made in our workshop, using a personal computer for data recording. 


\section{TABLE II}

\section{Kinetics of Hydrolysis of a Number of Defined Substrates by Human $\alpha$-Thrombin and Human Factor $\mathrm{Xa}$ in Buffer $\mathrm{A}$ at $37^{\circ} \mathrm{C}$.}

\begin{tabular}{|c|c|c|c|c|c|c|c|}
\hline \multirow[b]{2}{*}{ Entry } & \multirow[b]{2}{*}{ Substrate } & \multicolumn{3}{|c|}{ h- $\alpha$-thrombin } & \multicolumn{3}{|c|}{ h-factor $X \mathbf{a}$} \\
\hline & & $\begin{array}{l}K_{m} \\
\mu \mathrm{M}\end{array}$ & $\begin{array}{c}\mathbf{k}_{\text {cat }} \\
\mathrm{s}^{-1}\end{array}$ & $\begin{array}{c}\mathrm{k}_{\mathrm{cat}} / \mathrm{K}_{\mathrm{m}} \\
(\mathrm{Ms})^{-1}\end{array}$ & $\begin{array}{l}K_{m} \\
\mu M\end{array}$ & $\begin{array}{c}\mathbf{k}_{\mathrm{cat}} \\
\mathrm{s}^{-1}\end{array}$ & $\begin{array}{c}\mathbf{k}_{\mathrm{cat}} / \mathbf{K}_{\mathrm{m}} \\
(\mathrm{Ms})^{-1}\end{array}$ \\
\hline 1 & 2HCl.H-D-Phe-Pip-Arg-pNA $(1)^{\#}$ & 6 & 237 & 3.95E7 & 174 & 8 & $4.60 \mathrm{E} 4$ \\
\hline 2 & 2HCl.H-D-Phe-Pro-Arg-pNA (2) & 3 & 28 & 9.33E6 & 690 & 19 & $2.75 \mathrm{E} 2$ \\
\hline 3 & 2HCl.H-D-Phe-Pip-Lys-pNA (1a) & 65 & 118 & $1.82 \mathrm{E} 6$ & 960 & 0.6 & $6.25 \mathrm{E} 2$ \\
\hline 4 & 2HCl.H-D-Phe-Pro-Lys-pNA (2a) & 35 & 90 & $2.57 \mathrm{E} 6$ & - & - & - \\
\hline 5 & 2HCl.H-D-Phe-Pip-Orn-pNA (1b) & 420 & 5 & $1.19 \mathrm{E} 4$ & NH & - & - \\
\hline 6 & HCl.H-D-Phe-Pip-Nle-pNA (1d) & 943 & 11 & $1.17 \mathrm{E} 4$ & NH & - & - \\
\hline 7 & HCl.H-D-Phe-Pro-Nle-pNA (2b) & 88 & 7 & $7.95 \mathrm{E} 4$ & - & - & - \\
\hline 8 & HCl.H-D-Phe-Pip-Glu-pNA (1c) & 474 & 0.4 & $8.44 \mathrm{E} 2$ & NH & - & - \\
\hline 9 & 2HCl.H-Gly-Arg-pNA (3) & 2280 & 0.10 & $4.38 \mathrm{El}$ & - & - & - \\
\hline 10 & 2HCl.H-Ala-Arg-pNA (4) & 1140 & 0.07 & $6.14 \mathrm{EI}$ & - & - & - \\
\hline 11 & 2HCl.H-D-Ala-Arg-pNA (5) & 4100 & 0.10 & $2.44 \mathrm{El}$ & - & - & - \\
\hline 12 & 2HCl.H-Val-Arg-pNA (6) & 1240 & 0.23 & $1.85 E 2$ & NH & - & - \\
\hline 13 & 2HCl.H-D-Val-Arg-pNA (7) & 4250 & 0.3 & $7.06 \mathrm{EI}$ & - & $=$ & - \\
\hline 14 & MZ-Gly-Arg-pNA.HCI (3a) & 2540 & 12 & $4.72 \mathrm{E} 3$ & 4700 & 55 & $1.17 \mathrm{E} 4$ \\
\hline 15 & MZ-Ala-Arg-pN $\Lambda . \mathrm{HCl}(4 a)$ & 1540 & 108 & $7.0 \mathrm{lE} 4$ & 5600 & 52 & $9.29 \mathrm{E} 3$ \\
\hline 16 & MZ-D-Ala-Arg-pNA.HCl (5a) & 1330 & 0.06 & $4.5 \mathrm{IE} 1$ & 3100 & 0.09 & $2.90 \mathrm{El}$ \\
\hline 17 & MZ-Aib-Arg-pNA.HCl* (SQ68) & 830 & 0.46 & $5.54 \mathrm{E} 2$ & 3940 & 2.9 & $7.38 \mathrm{E} 2$ \\
\hline 18 & MZ-Val-Arg-pNA.HCl (6a) & 1000 & 14 & $1.40 \mathrm{E} 4$ & 2570 & 6 & $2.33 \mathrm{E} 3$ \\
\hline 19 & MZ-D-Val-Arg-pNA.HCl (7a) & 1310 & 0.15 & $1.15 \mathrm{E} 2$ & 1820 & 0.13 & 7.14E1 \\
\hline 20 & $\mathrm{MMZ}$-Gly-Arg-pNA.HCl (3b) & 1740 & 7 & $4.02 \mathrm{E} 3$ & 6400 & 44 & $6.88 \mathrm{E} 3$ \\
\hline 21 & MMZ-Ala-Arg-pNA.HCl (4b) & 670 & 17 & $2.54 \mathrm{E} 4$ & 7100 & 41 & $5.77 \mathrm{E} 3$ \\
\hline 22 & DMMZ-Gly-Arg-pNA.HCl (3c) & 900 & 6 & $6.67 \mathrm{E} 3$ & 2840 & 14 & 4.93E3 \\
\hline 23 & DMMZ-Ala-Arg-pNA.HCl (4c) & 570 & 4 & $7.02 \mathrm{E} 3$ & - & - & 4.93E $3^{* *}$ \\
\hline 24 & DEMZ-Gly-Arg-pNA.HCl (3d) & 870 & 2 & $2.30 \mathrm{E} 3$ & 2320 & 5 & $2.16 \mathrm{E} 3$ \\
\hline 25 & Nas-Gly-Arg-pNA.HCl (3e) & NH & - & - & 120 & 2 & $1.67 \mathrm{E} 4$ \\
\hline 26 & Tos-Gly-Arg-pNA.HCl (3f) & NH & - & - & 230 & 9 & $3.91 \mathrm{E} 4$ \\
\hline
\end{tabular}

NH: not hydrolyzed; " from reference $1 ;{ }^{\#}$ substrate numbering as given in table $\mathrm{I} ;{ }^{* *}$ non Michaelis-Menten kinetics.

In a polystyrene microcuvette (total volume $500 \mu \mathrm{L}$ ), buffer $A$ and substrate solution were added to obtain a final substrate concentration between 1 to $2000 \mu \mathrm{M}$. After 5 min of incubation at $37^{\circ} \mathrm{C}$, enzyme solution was added to achieve a final concentration between 0.5 to $100 \mathrm{nM}$. The measurement was carried out in a thermostated cuvette-holder at $37^{\circ} \mathrm{C}$. The Michaelis constant $\left(\mathrm{K}_{\mathrm{m}}\right)$ and the catalytic constant $\left(\mathrm{k}_{\text {cat }}\right)$ were obtained by measuring initial reaction velocities at different substrate concentrations (see table II). The data obtained were fitted using a non-linear regression procedure to the Michaelis-Menten equation.

Continuous registration of the thrombin generation curve: The measurement is described in full detail in reference 1 . Briefly, to $400 \mu \mathrm{L}$ defibrinated plasma in a polystyrene microcuvette was added $110 \mu \mathrm{L}$ buffer $\mathrm{A}, 20 \mu \mathrm{L}$ phospholipid solution (phosphatidyl choline/phosphatidyl serine $8: 2$, final concentration $1.5 \mu \mathrm{M}$ ) and $30 \mu \mathrm{L}$ substrate solution (final concentration $500 \mu \mathrm{M}$ ). After $4 \mathrm{~min}$ of incubation at $37^{\circ} \mathrm{C}, 20 \mu \mathrm{L}$ of a recombinant tissue factor solution (final concentration 6 $\mathrm{ng} / \mathrm{mL}$ ) was added. Thrombin generation was started by adding $20 \mu \mathrm{L}$ of a $0.5 \mathrm{M} \mathrm{CaCl}_{2}$ solution (final concentration $16.7 \mathrm{mM}$ ); during the measurement the temperature was kept at $37^{\circ} \mathrm{C}$. The 

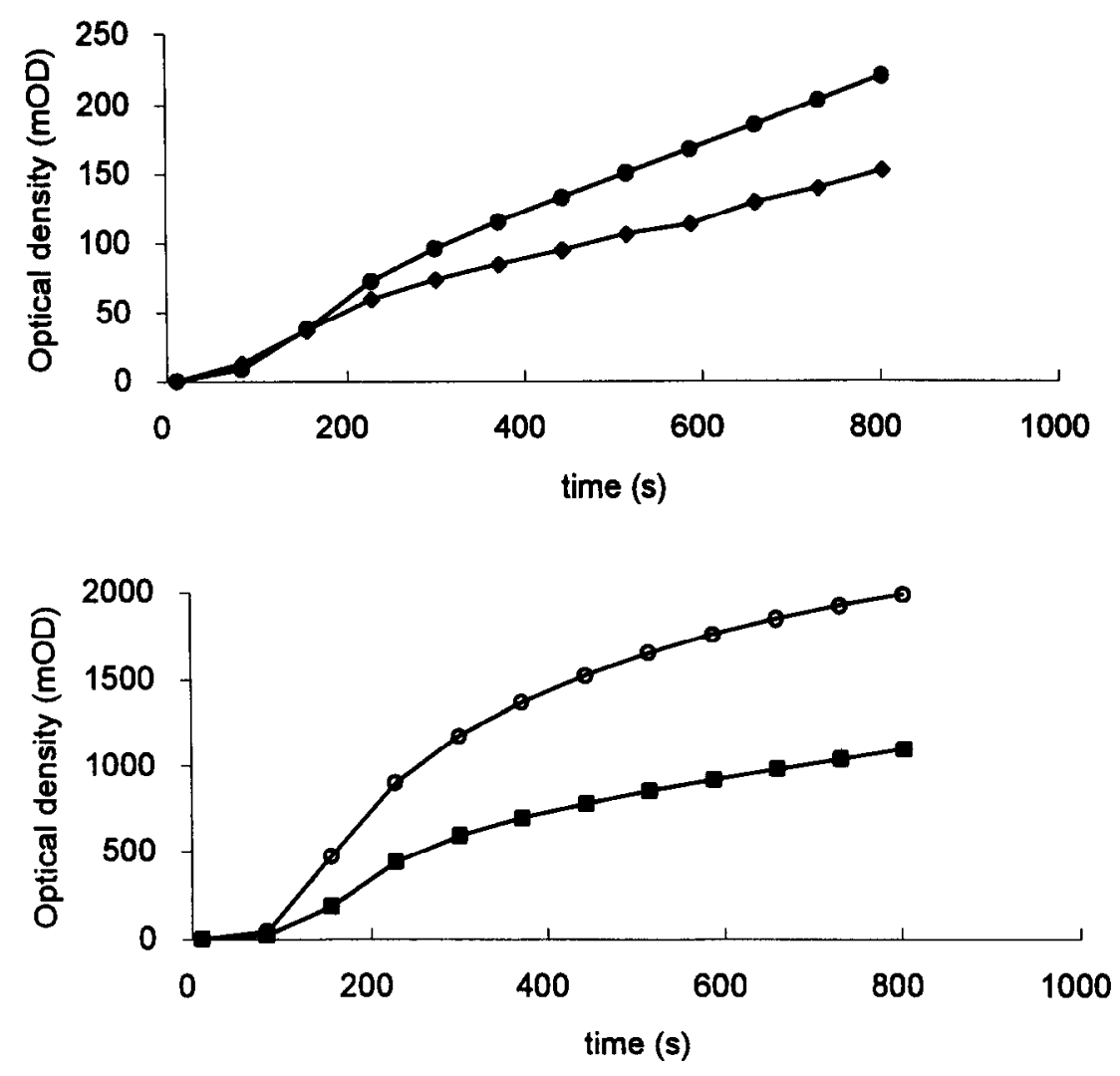

FIG. 1.

Continuous optical density curves measured with different substrates in tissue factor triggered plasma. Upper frame: $-2 \mathrm{HCl} . \mathrm{H}-\mathrm{Val}-\mathrm{Arg}-\mathrm{pNA}(\mathbf{6})$, $\bullet$ MZ-Aib-Arg-pNA.HCl (SQ68, reference); lower frame: O: 2HCl.H-D-Phe-Pip-Orn-pNA (1b), D. DEMZ-GlyArg-pNA.HCl (3d). The optical density curve measured with HCl.H-D-Phe-Pip-Glu-pNA (1c) is omitted for clarity because it overlaps the curve measured with SQ68. The optical density curve is the time integral of a thrombin generation curve. The final slope of this curve is the result of the amidolytic activity of the $\alpha_{2}$ macroglobulin-thrombin complex.

optical density was monitored at $405 \mathrm{~nm}$, see Fig. 1. From the optical density curve, the first derivative can be calculated giving the enzyme concentration during the measurement

\section{RESULTS AND CONCLUSION}

The thrombin generation curve is a rich source of information on the coagulation process in an individual plasma sample. With the usual subsampling techniques (22), the experimental effort to obtain such a curve prohibits its application to plasma samples of individual patients in the clinical laboratory. The continuous method published earlier (1), allows the registration of a thrombin generation curve in a single sample by optical monitoring of the thrombin-dependent splitting of a chromogenic substrate. This method lends itself to adaptation to laboratory automatons. It 
requires thrombin substrates that are not exhausted by the thrombin generation in a plasma sample. Current substrates such as S2238 (Kabi) do not fulfill this requirement but another substrate, SQ68 (Serbio) did. In search for specific thrombin substrates that are specific but nevertheless are converted slowly, a series of 25 peptide $p$-nitroanilides with H-D-Phe-Pip-ArgpNA and $\mathrm{CH}_{3} \mathrm{O}-\mathrm{CO}-\mathrm{CH}_{2}-\mathrm{CO}-\mathrm{Aib}-\mathrm{Arg}-\mathrm{pNA}$ as lead structures was synthesized. Classical solution methods were used and good yields were obtained.

Substrates containing the H-D-Phe-Pip(Pro) sequence are hydrolyzed by thrombin even when neutral or acidic amino acid side chains are present at position P1. However, the efficient binding interaction of H-D-Phe-Pip(Pro) with thrombin results in $\mathrm{K}_{\mathrm{m}}$ values that were too low for our purpose. Varying $\mathrm{P}_{2} / \mathrm{P}_{3}$ residues appear to be the method of choice to arrive at substrates with higher $K_{m}$ values. In this study the amino acid at $P 1$ was always an arginine residue, which ensures optimal interaction with the $S_{1}$ specificity pocket. The amino acid residue at P2 was systematically changed, incorporating: glycine, alanine and valine. This resulted in a gradual increasing occupancy of the hydrophobic binding pocket, leading to more important interaction and thus higher suitability for our purposes. The $\mathrm{P} 3$ modification was either omitted (a free $\mathrm{N}$ terminus) or modified by a malonic acid derivative in which the methylene protons were changed for methyl- or ethyl groups. This resulted in fairly apolar aliphatic side chains which do not interact optimally with the aryl-binding site of thrombin. To achieve better interaction with the aryl-binding site, the $\mathrm{N}$-terminus was modified by the introduction of an aromatic function. For this purpose the $p$-toluenesulfonyl- and $\beta$-naphthalenesulfonyl function were chosen (see table I). This however, did not result in compounds that could be hydrolyzed by thrombin. The similarity of compounds $3 \mathrm{e}$ and $3 \mathrm{f}$ to NAPAP suggests a similar binding mode in which the anilide bond points away from the Ser195 side chain.

In the continuous assay we need substrates which do not interact with factor Xa to minimize inhibition of thrombin formation and unwanted substrate hydrolysis by factor $\mathrm{Xa}$. As can be seen from table II, compounds 1b, 1c, 3d and 6 appear to be suitable to be used as substrates in the continuous thrombin assay. A detailed biochemical evaluation of the applicability of these substrates in the continuous thrombin assay will be published elsewhere.

\section{Abbreviations}

Aib: aminoisobutyric acid ( $\alpha$-methylalanine); DCC: N,N'-dicyclohexylcarbodiimide; DCU: N,N'dicyclohexylurea; DEMZ: diethylmalonic acid monomethyl ester; DIPEA: N,Ndiisopropylethylamine; DMF: N,N-dimethylformamide; DMML: dimethylmalonic acid monomethyl ester; EtOAc: ethylacetate; MMZ: methylmalonic acid monomethyl ester; $\mathrm{MZ}$ : malonic acid monomethyl ester; pNA: para-nitroaniline; Nas: 2-naphthalenesulfonyl; Nle: norleucine; NMM: N-methylmorpholine; Orn: ornithine; Pip: pipecolic acid (homoproline); Tris: tris(hydroxymethyl)aminomethane; Tos: 4-toluenesulfonyl; UV: ultra-violet.

\section{REFERENCES}

1. HEMKER, H.C., WIELDERS, S., KESSELS, H. and BÉGUIN, S. Continuous registration of thrombin generation in plasma, its use for the determination of the thrombin potential. Thromb. Haemost. 70, 617-624, 1993.

2. BAJUSZ, S., SZÉLL, E., BAGDY, D., BARABAS, E., HORVATH, G., DIOSZEGI, M., FITTLER, Z., SZABO, G., JUHASZ, A., TOMORI, E. and SZILAGYI, G. Highly active and selective anticoagulants: D-Phe-Pro-Arg- $\mathrm{H}$, a free tripeptide aldehyde prone to sponteneous inactivation, and its stable $N$-methyl derivative, D-Me-Phe-Pro-Arg-H. J. Med. Chem. 33, 1729$1735,1990$.

3. KETTNER, C. and SHAW, E. D-Phe-Pro-Arg- $\mathrm{CH}_{2} \mathrm{Cl}$ a selective affinity label for thrombin. Thromb. Res. 14, 969-973, 1979.

4. KETTNER, C., MERSINGER, L. and KNABB, R. The selective inhibition of thrombin by peptides of boroarginine. J. Biol. Chem. 265, 18289-18297, 1990. 
5. CHENG, L., GOODWIN, C.A., SCULLY, M.F., KAKKAR, V.V. and CLAESON, G. Synthesis and biological activity of ketomethylene pseudopeptide analogues as thrombin inhibitors. J. Med. Chem. 35, 3364-3369, 1992.

6. WANG, C.-L.J., TAYLOR, T.L., MICAL, A.J., SPITZ, S. and REILLY, T.M. Synthesis of phosphonopeptides as thrombin inhibitors. Tetrahedron Lett. 33, 7667-7670, 1992.

7. BAJUSZ, S., BARABAS, E., FEHÉR, A., HORVATH, G., SZÉLL, E. and BAGDY, D. Thrombin inhibition by tripeptide aldehydes derived from D-Phe-Pro-Arg-H by replacing Arg with neutral residues. In: Peptides 1992, Proc. of the 22nd European Peptide Symposium. C.H. Schneider and A.N. Eberle (eds.), pp 784-785, ESCOM Science Publishers B.V., Leiden, (1993). 8. ELGENDY, S., DEADMAN, J., PATEL, G., GREEN, D., CHINO, N., GOODWIN, C.A., SCULLY, M.F., KAKKAR, V.V. and CLAESON, G. New peptide boronic acid inhibitors of thrombin. Tetrahedron Lett. 33, 4209-4212, 1992.

9. CHENG, L., GOODWIN, C.A., SCULLY, M.F., KAKKAR, V.V. and CLAESON, G. Substrate-related phosphonopeptides, a new class of thrombin inhibitors. Tetrahedron Lett. 32, 7333-7336, 1991

10. BODE, W., MAYR, I., BAUMANN, U., HUBER, R., STONE, S.R. and HOFSTEENGE, J. The refined $1.9 \AA$ crystal structure of human $\alpha$-thrombin: interaction with D-Phe-Pro-Arg chloromethylketone and significance of the Tyr-Pro-Pro-Trp insertion segment. EMBO J. 8, 3467-3475, 1989.

11. BODE, W., TURK, D. and KARSHIKOV, A. The refined $1.9 \AA X$-ray crystal structure of DPhe-Pro-Arg-chloromethylketone-inhibited human $\alpha$-thrombin: Structurc analysis, overall structure, electrostatic properties, detailed active-site geometry and structure function relationships. Protein Sci. 1, 426-471, 1992.

12. Serbio Laboratories, France; European Patent 88400304.7.

13. MATSUZAKI, T., SASAKI, C. and UMEYAMA, H. X-ray analysis of a thrombin inhibitortrypsin complex. J. Biochem. 105, 949-952, 1989.

14. BODE, W., TURK, D. and STÜRZEBECHER, J. Geometry of binding of the benzamidineand arginine-based inhibitors $N \alpha$-(2-naphthyl-sulphonyl-glycyl)-DL-p-amidinophenylalanyl-piperidine (NAPAP) and $(2 R, 4 R)$-4-methyl-1-[No-(3-methyl-1,2,3,4-tetrahydro-8-quinoline-sulphonyl)-Larginyl)-2-piperidine carboxylic acid (MQPA) to human $\alpha$-thrombin. Eur. J. Biochem. 193, 175182,1990

15. MATSUZAKI, T., SASAKI, C. and UMEYAMA, H. A predicted tertiary structure of a thrombin inhibitor-trypsin complex explains the mechanisms of the selective inhibition of thrombin, factor Xa, plasmin and trypsin. J. Biochem. 103, 537-543, 1988.

16. TURK. D., STURZEBECHER J. and BODE, W. Geometry of binding of the No-tosylated piperidines of $m$-amidino-, $p$-amidino- and $p$-guanidino phenylalanine to thrombin and trypsin. $\mathrm{X}$ ray crystal structures of their trypsin complexes and modeling of their thrombin complexes. FEBS Letters 287, 133-138, 1991 .

17. BANNER, D. and HADVARY, P. Crystallographic analysis at $3.0 \AA$ resolution of the binding to human thrombin of four active site-directed inhibitors. J. Biol. Chem. 266, 20085-20093, 1991.

18. BRANDSTETTER, H., TURK, D., HOEFFKEN, H.W., GROSSE, D., STÜRZEBECHER, J., MARTIN, P.D., EDWARDS, B.F.P. and BODE, W. Refined 2.3 $\AA$ X-ray crystal structure of bovine thrombin complexes formed with the benzamidine and arginine-based thrombin inhibitors NAPAP, 4-TAPAP and MQPA. J. Mol. Biol. 226, 1085-1099, 1992.

19. SCHNABEL, E. Verbesserte Synthese von tert.-Butyloxycarbonyl-aminosäuren durch $\mathrm{pH}-$ Stat-Reaktion. Liebigs Ann. Chem. 702, 188-196, 1967.

20. RIJKERS, D.T.S., HEMKER, H.C., NEFKENS, G.H.L. and TESSER, G.I. The use of phosphorus oxychloride in the synthesis of amino acid $p$-nitroanilides. Recl. Trav. Chim. PaysBas 110,347-348, 1991

21. BRENNER, M. and HUBER, W. Herstellung von $\alpha$-Aminosäureestern durch Alkoholyse der Methylester. Helv. Chim. Acta 36, 1109-1115, 1953.

22. HEMKER, H.C., WILLEMS, G.M. and BÉGUIN, S. A computer assisted method to obtain the prothrombin activation velocity in whole plasma independent of thrombin decay processes. Thromb. Haemost. 56, 9-17, 1986. 